BULL. AUSTRAL. MATH. SOC.

\title{
TENSOR PRODUCTS OF FUNCTION ALGEBRAS
}

\author{
Athanasios KYRIAZIS
}

For appropriate topclogical spaces $X, Y, Z$ the algebra $\mathcal{C}_{c}\left(X \times{ }_{Z} Y\right)$ of $\mathbb{Z}$-valued continuous functions on the fibre product $X{ }_{Z}{ }_{Y}$ in the compact-open topology, describes the completed biprojective $C_{c}(Z)$-tensor product of $C_{c}(X), C_{c}(Y)$.

The purpose of this note is to show the following:

THEOREM. Let $X, Y, Z$ be completely regular spaces with $X, Y$ o-compact. Moreover, let $X \times{ }_{Z} Y$ be the fibre product of $X, Y$ over $Z$. Then,

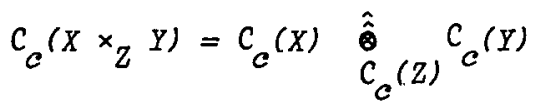

within an isomorphism of Frechet locally m-convex $C_{c}(2)$-algebras.

Concerning the definition of the topological $c_{c}(2)$-algebra in the second member of (1) see [5: Definition 1.1 and also (1.6)]. Relations analogous to (II are valid too for algebras of complex-valued holomorphic functions on stein manifolds and $c^{\infty}$-functions on compact $c^{\infty}$-manifolds (see Scholium).

Received 18 November 1986. $\$ \mathrm{~A} 2.00+0.00$.

Copyright Clearance Centre, Inc. Serial-fee code: 0004-9727/87 
We first comment on the necessary terminology. Thus let $X, Z$ be topological spaces and $\mu: X \rightarrow Z$ a continuous map. The algebra $C_{c}(X)$ of $\mathbb{C}$-valued continuous functions on $X$ with the compact-open topology becomes a locally m-convex $\mathcal{C}_{C}(Z)$-algebra via a " $\mu$-convolution" given by

$$
a_{*_{\mu}} f:=(a \circ \mu) \cdot f
$$

for $a \in C_{c}(Z), f \in C_{c}(X)$ (see [5: Section 1]).

On the other hand, we say that a topological algebra $M$ admits a functional representation whenever one has $C_{c}(M(M))=M$, within an isomorphism of topological algebras [1 : p.474, Theorem 3.1]. So first we have.

LEMMA 1. Let $X, Y, Z$ be completely regular spaces and $X x_{Z} Y$ the fibre product of the maps $\mu: X \rightarrow Z$ and $\nu: Y \rightarrow Z$. Then, in the category of topological algebras admitting functional representations, the algebra $\mathrm{C}_{c}\left(X \times_{Z} Y\right)$ is the pushout of the maps $\mu_{*}: C_{c}(Z)+C_{c}(X)$ and $\nu_{*}: C_{c}(Z)+C_{c}(Y)$ defined by $\mu_{*}(a):=a \circ \mu_{,} v_{*}(a):=a \circ v\left(a \in C_{c}(Z)\right)$.

Proof. Let $\tilde{p}, \tilde{q}$ be the canonical projections of $X \times{ }_{Z} Y:=\{(x, y) \in X \times Y: \mu(x)=v(y)\}$ onto $X, Y$ respectively. Then, one has

$$
\mu \circ \tilde{p}=\nu \circ \tilde{q}
$$

such that the following diagram is comutative

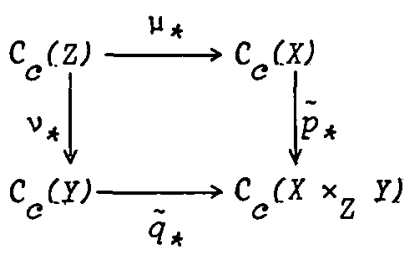

Moreover, let $(M, r, s)$ be a triad consisting of a locally $m$-convex algebra $M$ admitting a functional representation and continuous algebra morphisms 
$r: C_{c}(X) \rightarrow M, s: C_{c}(Y) \rightarrow M$ such that

$$
r \circ \mu_{*}=\varepsilon \circ \nu_{*}
$$

The "transpose" continuous maps of $r, s$ on the spectra of the respective algebras, that is $r^{*}: M(M) \rightarrow M\left(C_{c}(X)\right), s^{*}: M(M) \rightarrow M\left(C_{c}(Y)\right)$ make the next diagram commutative.

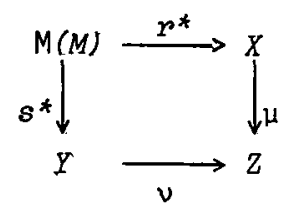

(see also [6:p.223, Theorem 1.2]). Thus, there exists a unique continuous map

$$
\psi: M(M) \rightarrow X \times_{Z} Y
$$

such that

$$
\tilde{p} \circ \psi=r^{*}, \tilde{q} \circ \psi=s^{*}
$$

(see [8 : p.231, Definition 12]). Hence, one gets a continuous algebra morphism

$$
\psi_{*}: C_{c}\left(X \times_{Z} Y\right)+M
$$

such that

$$
\psi_{*} \circ \tilde{p}_{*}=r, \psi_{*} \circ \tilde{q}_{*}=\boldsymbol{s},
$$

which yields the assertion (see [8 : p.255, Definition 10]).

Now, if $E$ is a topological algebra and $I$ a closed 2-sided ideal of $E$, one has $M(E / I)=h(I)$, within a homeomorphism. Here $h(I)=\{f \in M(E): I \subseteq \operatorname{ker}(f)\}$ denotes the hull of $I$ (see $[6:$ p.339, Theorem 4.1]). This yields the following

LEMMA 2. Let $E$ be a topological algebra admitting a functional representation and $I$ a closed 2-sided ideal of $E$. Then, the quotient topological algebra $E / I$ admits a functional representation. 
Let $X, Y, Z$ be completely regular spaces and $J$ the closed subspace of $C_{c}(X \times Y)$ generated by the set $T:=\left\{a{ }_{\mu}^{*} f-a{ }_{\nu}^{*} f: a \in C_{c}(Z)\right.$ $\left.f \in C_{c}(X \times Y)\right\}$ (see (2)). Then, $J$ is a closed 2-sided $C_{c}(Z)$-ideal of $C_{c}(X \times Y)$ (see (2) and also [5: Section 1]), such that $C_{c}(X \times Y) / J$ is a locally m-convex $c_{c}(Z)$-algebra (see $\left.[5],[6]\right)$. Moreover, the homeomorphism $M\left(C_{c}(X \times Y)\right)=X \times Y \quad$ (see $[6: \mathrm{p} .223$, Theorem 1.2]) and Lemma 2 imply the following

COROLLARY 1. If $X, Y$ are completely regular spaces, the algebra $C_{c} C X \times I L / J$ admits a functional representation.

LEMMA 3. Let $X, Y, \dot{Z}$ be completely regular spaces and $X \times_{Z} Y$ the fibre product of the mops $\mu: X \rightarrow Z$ and $\nu: Y \rightarrow Z$. Then, the algebra $\mathcal{C}_{c}(X \times Y) / J$ is the pushout of $C_{c}(X), C_{c}(Y)$ over $C_{c}(Z)$ in the category of topological algebras admitting functional representations.

Proof. If $p, q$ are the canonical projections of $X \times Y$ onto $X, Y$ respectively, then Corollary 1 and (5) imply

$$
\hat{p}_{*} \circ \mu_{*}=\hat{q}_{*} \circ v_{*}
$$

where $\hat{p}_{*}, \hat{q}_{*}$ are the compositions of the continuous algebra morphisms

$$
\begin{aligned}
& C_{c}(X) \underset{P_{*}}{\longrightarrow} C_{c}(X \times Y) \longrightarrow \underset{c}{\longrightarrow} C_{c}(X \times Y) / J \\
& c_{c}(Y) \underset{q_{*}}{\longrightarrow} c_{c}(X \times Y) \longrightarrow{ }_{c}(X \times Y) / J
\end{aligned}
$$

respectively (see Lemma 1). Moreover, let $(M, r, s)$ be a system consisting of a locally m-convex algebra $M$ adritting a functional representation and continuous algebra morphisms $r, s$ satisfying (5). Thus, the (uniquely definedl continuous map (7) implies the existence of a continuous map

$$
\psi^{\prime}: M(M) \rightarrow X \times Y
$$

such that $p \circ \psi^{\prime}=p^{*}, q^{\circ} \psi^{\prime}=8^{*}$. Hence, one gets a continuous algebra morphism 


$$
\psi_{*}^{\prime}: \mathrm{C}_{c}(X \times Y) \rightarrow M
$$

with $\psi_{*}^{\prime} \circ p_{*}=r_{3} \psi_{*}^{\prime} \circ q_{*}=s$ (see (12), (8), (10)). Furthermore, the relation $J=\operatorname{ker}(\pi) \subseteq \operatorname{ker}\left(\psi_{*}^{\prime}\right) \quad$ (see Corollary 1, (12), (14)) implies the existence of a (unique) continuous algebra morphism

$$
\hat{\psi}: C_{c}(X \times Y) / J \rightarrow M
$$

with $\psi_{*}^{\prime}=\hat{\psi} \circ \pi$. Therefore, one gets $r=\hat{\psi} \circ \hat{p}_{*}, s=\hat{\psi} \circ \hat{q}_{*}$ (see (14), (11), (15)), and the assumption follows [8: p.255, Definition 10].

Now, by the uniqueness of the pushout in a given category $[8: p .255]$ in connection with Lemmas 1,3 one gets the following

PROPOSITION. Let $X, Y, Z$ be completely regular spaces and $X \times{ }_{Z} Y$ the fibre product of $X, Y$ over $Z$ (see Lemma 1). Then,

$$
C_{c}\left(X \times{ }_{Z} Y\right)=C_{c}(X \times Y) / J
$$

within an isomorphism of locally m-convex algebras. In particular, (16) yields an isomorphism of locally m-convex $C_{c}(Z)$-algebras (see (2)).

Let $X, Y$ be completely regular $k$-spaces with $X \times Y$ a $k$-space as well (take, for example, $X, Y$ to be locally compact spaces; see [1]). Thus one has the following isomorphism of locally $m$-convex algebras.

$$
c_{c}(X) \hat{\hat{\theta}} c_{c}(Y)=c_{c}(X \times Y)
$$

(see [6 : p.392. Corollary 1.1]). Moreover, if $Z$ is a completely regular space, (17) preserves the respective topological $\mathcal{C}_{c}(Z)$-algebra structures (see (2ll, such that (17) yields an isomorphism of locally $m$-convex $\mathcal{C}_{c}(Z)$-algebras. Furthermore, let $I$ be the closed 2-sided $c_{c}(Z)$-ideal of $C_{c}(X) \otimes C_{c}(Y)$ defined by the set $S:=\left\{\left(a *_{\mu} f\right) \otimes g-f \otimes\left(a{ }^{*} g\right)\right.$ : $\left.a \in \mathcal{C}_{c}(Z), f \in \mathcal{C}_{c}(X), g \in \mathcal{C}_{c}(Y)\right\}$. BY (17) $I$ is a dense subset of $J$ (see corollary 1), hence

$$
C_{c}(X) \hat{\theta} C_{c}(Y) / \hat{I}=C_{c}(X \times Y) / J
$$


within an isomorphism of (complete) locally m-convex $C_{c}(Z)$-algebras.

We are now in the position to give the

Proof of Theorem. We first remark that $\mathcal{C}_{c}(X) \hat{\hat{\theta}} \mathcal{C}_{c}(y)$ is a Fréchet locally m-convex $C_{c}(Z)$-algebra (see $[6:$ p.392, Corollary 1.1], $[3:$ p.345, Proposition 2] and (17)). Hence $([5:(1.6)]$ and $[2:$ p.113 and also p.138, Theorem 2]) one has the next topological-algebraic isomorphism

$$
C_{c}(X) \hat{\hat{\theta}}_{C_{c}(Z)} C_{c}(Y)=C_{c}(X) \hat{\hat{\theta}} C_{c}(Y) / \hat{I}
$$

such that the assumption now follows from (18) and the Proposition.

SCHOLIUM. We get relations analogous to (1) by considering (complexvalued) holomorphic and $C^{\infty}$-functions. Thus, if $X, Y, Z$ are $s$ tein spaces [4], the fibre product $X \times_{Z} Y$ is a Stein space [4: p.225, E.51b]; so we get results analogous to Lemas 1,3 in the category of Stein algebras (see also [6: p.229; (3.2)] for this type of algebras). Thus, one has

$$
O\left(X \times_{Z} Y\right)=O(X) \hat{\hat{\theta}}_{O(Z)} O(Y)
$$

within an isomorphism of Frechet locally $m$-convex algebras (see the Proposition and $[6:$ p.402; (4.10)].

Moreover, suppose that $X, Y, Z$ are compact $C^{\infty}$-manifolds, with $X \times{ }_{Z} Y$ a (compact) $C^{\infty}$-manifold too (this happens, for example, if one of the $C^{\infty}$-functions $\mu, \nu$ (see Lema 1$)$ is a submersion). Hereafter $C^{\infty}(X)$ stands for the algebra of complex-valued $C^{\infty}$-functions on a smooth manifold $X$ endowed with the (canonical) $C^{\infty}$-topology (see for example [6 : Chapter IV, 4.(2)]). Thus consider now topological algebras "admitting differentiable representations"; that is, topological algebras $M$ such that $C^{\infty}(M(M))=M$, within a topological-algebraic isomorphism (see [6:p.227, Theorem 2.1]). So, by adapting Lemmas 1,3 and then the Proposition and Theorem, we have 


$$
C^{\infty}\left(X \times_{Z} Y\right)=C^{\infty}(X) \hat{\hat{\theta}}_{C^{\infty}(Z)} C^{\infty}(Y)
$$

within an isomorphism of Fréchet locally m-convex $\mathcal{C}^{\infty}(Z)$-algebras.

\section{References}

[1] J. Dugundji, Topology, (Allyn and Bacon, Boston, 1970).

[2] J. Horvath, Topological vector spaces and distributions, I, (Addison-Wesley, Reading. Mass., 1966).

[3] H. Jarchow, Locally convex spaces, (B.G. Teubner, Stuttgart, 1981).

[4] L. Kaup and B. Kaup, Holomorphic functions of several variables, (Walter de Gruyter, Berlin, 1983).

[5] A. Kyriazis, "On the spectra of topological A-tensor product A-algebras", Yokohama Math. J. 31 (1983), 47-65.

[6] A. Mallios, Topological algebras. Selected topics, (North-Holland, Amsterdam, 1986).

[7] A. Mallios, on functional representations of topological algebras", J. Funct. Anal. 6 (1970), 468-480.

[8] A. Solian, Theory of modules, (J. Wiley and Sons, N.Y., 1977).

Mathematical Institute

University of Athens

57, Solonos Street,

Athens 106 79,

Greece 